\title{
Partial caging: a clearance-based definition, datasets, and deep learning
}

\author{
Michael C. Welle ${ }^{1}$ (D) $\cdot$ Anastasiia Varava $^{1} \cdot$ Jeffrey Mahler $^{2} \cdot$ Ken Goldberg $^{2} \cdot$ Danica Kragic $^{1} \cdot$ Florian T. Pokorny $^{1}$
}

Received: 26 February 2020 / Accepted: 13 January 2021 / Published online: 4 February 2021

(c) The Author(s) 2021

\begin{abstract}
Caging grasps limit the mobility of an object to a bounded component of configuration space. We introduce a notion of partial cage quality based on maximal clearance of an escaping path. As computing this is a computationally demanding task even in a two-dimensional scenario, we propose a deep learning approach. We design two convolutional neural networks and construct a pipeline for real-time planar partial cage quality estimation directly from 2D images of object models and planar caging tools. One neural network, CageMaskNN, is used to identify caging tool locations that can support partial cages, while a second network that we call CageClearanceNN is trained to predict the quality of those configurations. A partial caging dataset of 3811 images of objects and more than 19 million caging tool configurations is used to train and evaluate these networks on previously unseen objects and caging tool configurations. Experiments show that evaluation of a given configuration on a GeForce GTX 1080 GPU takes less than $6 \mathrm{~ms}$. Furthermore, an additional dataset focused on grasp-relevant configurations is curated and consists of 772 objects with 3.7 million configurations. We also use this dataset for 2D Cage acquisition on novel objects. We study how network performance depends on the datasets, as well as how to efficiently deal with unevenly distributed training data. In further analysis, we show that the evaluation pipeline can approximately identify connected regions of successful caging tool placements and we evaluate the continuity of the cage quality score evaluation along caging tool trajectories. Influence of disturbances is investigated and quantitative results are provided.
\end{abstract}

Keywords Deep learning in robotics · Grasping - Computational geometry · Topological representation and abstraction of configuration spaces

\section{Introduction}

This is one of the several papers published in Autonomous Robots comprising the Special Issue on Topological Methods in Robotics.

Michael C. Welle

mwelle@kth.se

Anastasiia Varava

varava@kth.se

Jeffrey Mahler

jmahler@berkeley.edu

Ken Goldberg

goldberg@berkeley.edu

Danica Kragic

dani@kth.se

Florian T. Pokorny

fpokorny@kth.se

1 KTH Royal Institute of Technology, Stockholm, Sweden

2 University of California, Berkeley, USA
A rigid object is caged if it cannot escape arbitrarily far from its initial position. From the topological point of view, this can be reformulated as follows: an object is caged if it is located in a bounded connected component of its free space. This notion provides one of the rigorous paradigms for reasoning about robotic grasping besides form and force closure grasps (Bicchi and Kumar 2000; Rodriguez et al. 2012). While form and force-closure are concepts that can be analyzed in terms of local geometry and forces, the analysis of caging configurations requires knowledge about a whole connected component of the free configuration space and is hence a challenging problem that has been extensively studied analytically. However, since global properties of configuration space may also be estimated more robustly than subtle local geometric features used in classical force closure analysis, caging may hold promise particularly as a noise-tolerant approach to grasping and manipulation. 
In its topological formulation, caging is closely related to another global characteristic of configuration spaces-pathconnectedness, and, in particular, is a special case of the path non-existence problem (McCarthy et al. 2012; Varava et al. 2018). This is a challenging problem, as it requires reasoning about the entire configuration space, which is currently not possible to reconstruct or approximate (McCarthy et al. 2012; Varava et al. 2018).

Another interesting global characteristic of a configuration space is the maximum clearance of a path connecting two points. In path planning, paths with higher clearance are usually preferred for safety reasons. In contrast, in manipulation, if an object can escape from the manipulator only through a narrow passage, escaping is often less likely. In practical applications, it might be enough to partially restrict the mobility of the object such that it can only escape through narrow passages instead of completely caging it. Such configurations are furthermore less restrictive than full cages, thus allowing more freedom in placing caging tools.

This reasoning leads to the notion of partial caging. This generalization of classical caging was first introduced by Makapunyo et al. (2012), where the authors define a partial caging configuration as a non-caging formation of fingers that only allows rare escape motions. While Mahler et al. (2016) and Mahler et al. (2018) define a similar notion as energy-bounded caging, we propose a partial caging quality measure based on the maximum clearance along any possible escaping path. This value is directly related to the maximum width of narrow passages separating the object from the rest of the free space. Assuming motion is random, the quality of a partial cage depends on the width of a "gate" through which the object can escape.

Our quality measure is different from the one proposed in Makapunyo et al. (2012), where the authors introduced a measure based on the complexity and length of paths constructed by a sampling-based motion planner, thus generalizing the binary notion of caging to a property parameterized by cage quality.

One challenge with using sampling-based path planners for partial caging evaluation is that a single configuration requires multiple runs of a motion planner and-in the case of rapidly exploring random tree (RRT)—potentially millions of tree expansion steps each, due to the non-deterministic nature of these algorithms. This increases the computation time of the evaluation process which can be critical for realtime applications, such as scenarios where cage quality needs to be estimated and optimized iteratively to guide a caging tool from a partial towards a final cage. We significantly speed up the evaluation procedure for partial caging configurations by designing a deep learning-based pipeline that identifies partial caging configurations and approximates the partial caging evaluation function (we measured an evaluation time of less than $6 \mathrm{~ms}$ for a single given configuration

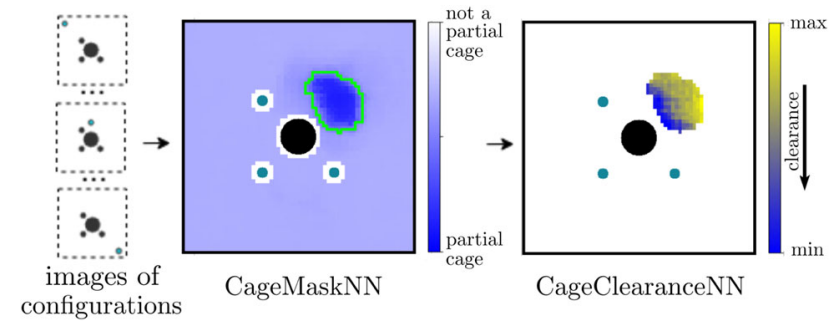

Fig. 1 Given an image of an object (depicted in black) and 3 or 4 caging tools (depicted in green), CageMaskNN determines whether a configuration belongs to the "partial cage" subset. If it does, CageClearanceNN, evaluates its quality according to the clearance measure learned by the network. On the figure, the blue region corresponds to successful placements of the fourth finger according to CageMaskNN, and their quality predicted by CageClearanceNN (Color figure online)

on a GeForce GTX 1080 GPU). For this purpose, we create a dataset of 3811 two-dimensional object shapes and 19055000 caging tool configurations and use it to train and evaluate our pipeline.

Apart from evaluating given partial caging configurations, we also use the proposed quality measure to choose potentially successful placements of 1 out of 3 or 4 caging tools, assuming the positions of the remaining tools are fixed. In Fig. 1, we represent the output as a heat map, where for every possible translational placement of a caging tool along a grid the resulting partial caging quality value is computed. Another application of the pipeline is the evaluation and scoring of caging configurations along a given reference trajectory.

Furthermore, we explore different shape similarity measures for objects and evaluate them from the partial caging perspective. We propose a way to generate partial caging configurations for previously unseen objects by finding similar objects from the training dataset and applying partial caging configurations that have good quality score for these objects. We compare three different definitions of distance in the space of shapes: Hausdorff, Hamming, and the distance in the latent space of a variational autoencoder (VAE) trained on a set of known objects. Out experiments show that Hamming distance is the best at capturing geometric features of objects that are relevant for partial caging, while the VAEinduced distance has the advantage of being computationally efficient.

This paper is a revised and extended version of our previously published conference submission (Varava et al. 2019). The contribution of the extension with respect to the conference paper can be summarized as follows:

1. We define a grasping band for planar objects-the area around the object that is suitable for placing caging tools, 
created a new dataset ${ }^{1}$ consisting of partial caging configurations located in the grasping band;

2. We approximate our partial caging quality measure with a deep neural network trained on this new dataset;

3. We perform ablation studies to evaluate our deep network architecture;

4. We evaluate the adequacy of our partial caging quality measure by modeling the escaping process as a random walk, and measuring the escape time;

5. We propose a cage acquisition method for novel objects based on known partial caging configurations for similar objects; for this, we explore several different distance metrics;

6. We further evaluate the robustness of the cage acquisition with respect to noise.

\section{Related work}

One direction of caging research is devoted to point-wise caging, where a set of points (typically two or three) represents fingertips, and an object is usually represented as a polygon or a polyhedron, an example of a $2 \mathrm{D}$ cage can be seen in Fig. 2 on the left-hand side. Rimon and Blake in their early work (Rimon and Blake 1999) proposed an algorithm to compute a set of configurations for a two-fingered hand to cage planar non-convex objects. Later, Pipattanasomporn and Sudsang (2006) proposed an algorithm reporting all twofinger caging sets for a given concave polygon. Vahedi and van der Stappen (2008) described an algorithm that returns all caging placements of a third finger when a polygonal object and a placement of two other fingers are provided. Later, Rodriguez et al. (2012) considered caging as a prerequisite for a form closure grasp by introducing a notion of a pregrasping cage. Starting from a pregrasping cage, a manipulator can move to a form closure grasp without breaking the cage, hence guaranteeing that the object cannot escape during this process.

One can derive sufficient caging conditions for caging tools of more complex shapes by considering more complex geometric and topological representations. For example, an approach towards caging 3D objects with 'holes' was proposed by some of the authors in Pokorny et al. (2013), Stork et al. $(2013 b, a)$. Another shape feature was later proposed in Varava et al. (2016), where we presented a method to cage objects with narrow parts as seen in Fig. 2 on the right-hand side. Makita and Maeda (2008) and Makita et al. (2013) have proposed sufficient conditions for caging objects corresponding to certain geometric primitives.

Finally, research has studied the connectivity of the free space of the object by explicitly approximating it.

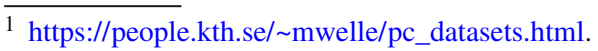
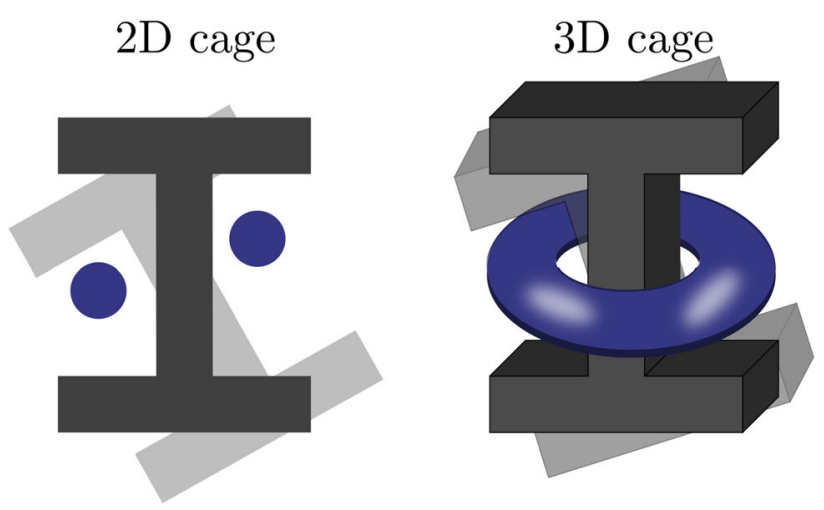

Fig. 2 Example of a 2D cage (left) and a 3D cage exploiting a narrow part of the object

For instance, Zhang et al. (2008) use approximate cell decomposition to check whether pairs of configurations are disconnected in the free space. Another approach was proposed by Wan and Fukui (2018), who studied cell-based approximations of the configuration space based on sampling. McCarthy et al. (2012) proposed to randomly sample the configuration space and reconstruct its approximation as a simplicial complex. Mahler et al. $(2016,2018)$ extend this approach by defining, verifying and generating energybounded cages - configurations where physical forces and obstacles complement each other in restricting the mobility of the object. These methods work with polygonal objects and caging tools of arbitrary shape, and therefore are applicable to a much broader set of scenarios. However, these approaches are computationally expensive, as discretizing and approximating a three-dimensional configuration space is not an easy task.

To enable a robot to quickly evaluate the quality of a particular configuration and to decide how to place its fingers, we design, train and evaluate a neural network that approximates our caging evaluation function (see Bohg et al. 2013 for an overview of data-driven grasping). This approach is inspired by recent success in using deep neural networks in grasping applications, where a robot policy to plan grasps is learned on images of target objects by training on large datasets of images, grasps, and success labels. Many experiments suggest that these methods can generalize to a wide variety of objects with no prior knowledge of the object's exact shape, pose, mass properties, or frictional properties (Kalashnikov et al. 2018; Mahler and Goldberg 2017; Zeng et al. 2017). Labels may be curated from human labelers (Kappler et al. 2015; Lenz et al. 2015; Saxena et al. 2008), collected from attempts on a physical robot (Levine et al. 2018; Pinto and Gupta 2016), or generated from analysis of models based on physics and geometry (Bousmalis et al. 2018; Gualtieri et al. 2016; Johns et al. 2016; Mahler et al. 2017). We explore the latter approach, developing a data-driven partial caging eval- 
uation framework. Our pipeline takes images of an object and caging tools as input and outputs $(i)$ whether a configuration is a partial cage and ( $i$ ) for each partial caging configuration, a real number corresponding to a predicted clearance, which is then used to rank the partial caging configuration.

Generative approaches to training dataset collection for grasping typically fall into one of three categories: methods based on probabilistic mechanical wrench space analysis (Mahler et al. 2017), methods based on dynamic simulation (Bousmalis et al. 2018; Johns et al. 2016), and methods based on geometric heuristics (Gualtieri et al. 2016). Our work is related to methods based on grasp analysis, but we derive a partial caging evaluation function based on caging conditions rather than using mechanical wrench space analysis.

\section{Partial caging and clearance}

\subsection{Partial caging}

In this section, we discuss the notion of partial caging defined in Varava et al. (2019). Let $\mathcal{C}$ be the configuration space of the object, ${ }^{2} \mathcal{C}_{\text {col }} \subset \mathcal{C}$ be its subset containing configurations in collision, and let $\mathcal{C}_{\text {free }}=\mathcal{C}-\mathcal{C}_{\text {col }}$ be the free space of the object. Let us assume $\mathcal{C}_{c o l}$ is bounded. Recall the traditional definition of caging:

Definition 1 A configuration $c \in \mathcal{C}_{\text {free }}$ is a cage if it is located in a bounded connected component of $\mathcal{C}_{\text {free }}$.

In practical applications, it may be beneficial to identify not just cages, but also configurations which are in some sense 'close' to a cage, i.e., configurations from which it is difficult but not necessarily impossible to escape. Such partial caging can be formulated in a number of ways: for example, one could assume that an object is partially caged if its mobility is bounded by physical forces, or it is almost fully surrounded by collision space but still can escape through narrow openings.

We introduce the maximal clearance of an escaping path as a quality measure. Intuitively, we are interested in partial caging configurations where an object can move within a connected component, but can only escape from it through a narrow passage. The 'width' of this narrow passage then determines the quality of a configuration.

Let us now provide the necessary definitions. Since, by our assumption, the collision space of the object is bounded, there exists a ball $B_{R} \subset \mathcal{C}$ of a finite radius containing it. Let us define the escape region $X_{\text {esc }} \subset \mathcal{C}$ as the complement of this ball: $X_{\text {esc }}=\mathcal{C}-B_{R}$.

\footnotetext{
${ }^{2}$ Note that in this paper we focus on the case where $\mathcal{C} \subset S E(2)$, but the definition of partial caging holds for arbitrary configuration spaces
}
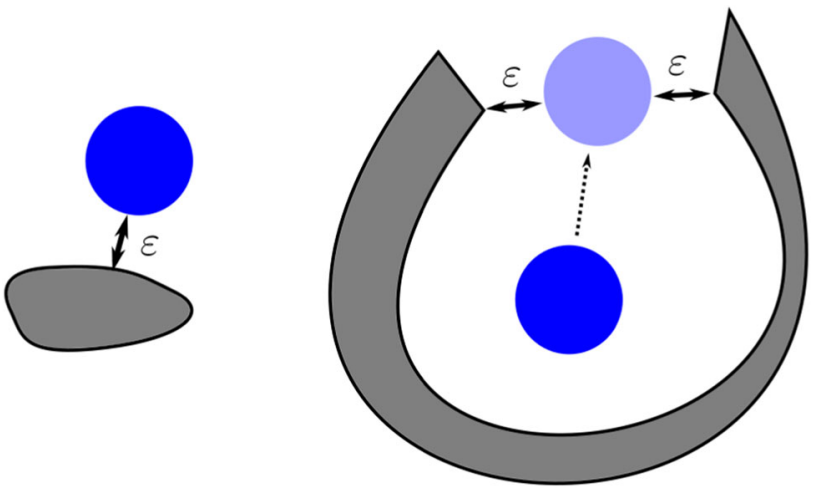

Fig. 3 On the left, an object (blue) can easily escape from the caging tool (grey); on the right, the object is partially surrounded by the caging tool and escaping is therefore harder. Both escaping paths will have the same clearance $\varepsilon$ (Color figure online)

Definition 2 A collision-free path $p:[0,1] \rightarrow \mathcal{C}_{\text {free }}$ from a configuration $c$ to $X_{e s c}$ is called an escaping path. The set of all possible escaping paths is denoted by $\mathcal{E P}\left(\mathcal{C}_{\text {free }}, c\right)$.

Let $c l: \mathcal{E P}\left(\mathcal{C}_{\text {free }}, c\right) \rightarrow \mathbb{R}_{+}$be a cost function defined as the minimum distance from the object along the path $p$ to the caging tools: $c l(p)=\min _{c \in p}\left(\operatorname{dist}\left(o_{c}, \mathbf{g}\right)\right)$ where $o_{c}$ is the object placed in the configuration $c$ and $\mathbf{g}$ denotes the caging tools. We define the caging evaluation function as follows:

$Q_{c l}(c)=\left\{\begin{array}{l}\min _{p \in \mathcal{E} \mathcal{P}\left(\mathcal{C}_{\text {free }}, c\right)} c l(p), \mathcal{E P}\left(\mathcal{C}_{\text {free }}, c\right) \neq \varnothing \\ 0, \mathcal{E} \mathcal{P}\left(\mathcal{C}_{\text {free }}, c\right)=\emptyset\end{array}\right.$

\subsection{The set $\mathcal{C}_{\text {cage }}$}

Observe that a low value of clearance measure on arbitrary configurations of $\mathcal{C}_{\text {free }}$ does not guarantee that a configuration is a sufficiently "good" partial cage. For example, consider only one convex caging tool located close to the object as in Fig. 3 (left). In this case, the object can easily escape. However, the clearance of this escaping path will be low, because the object is initially located very close to the caging tool. The same clearance value can be achieved in a much better partial caging configuration, see Fig. 3 (right). Here, the object is almost completely surrounded by a caging tool, and it can escape through a narrow gate. Clearly, the second situation is much preferable from the caging point of view. Therefore, we would like to be able to distinguish between these two scenarios.

Assume that caging tools are placed such that the object can escape. We increase the size of the caging tools by an offset, and eventually, for a sufficiently large offset, the object collides with the enlarged caging tools; let us assume that the size of the offset at this moment is $\varepsilon_{\text {col }}>0$. We are interested in those configurations for which there exists an intermediate 


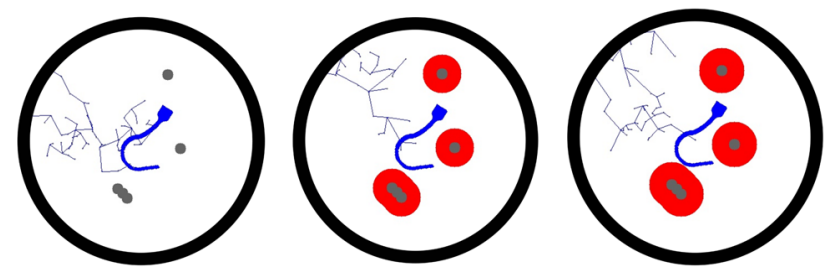

Fig. 4 The object (hook) is shown in blue while the caging tools are gray, the red symbolises the enlargement of the caging tools. The RRT nodes and edges are depicted in purple. From left to right, three enlargements of the caging tools are depicted. The object can always escape until its initial configuration stops being collision-free (Color figure online)

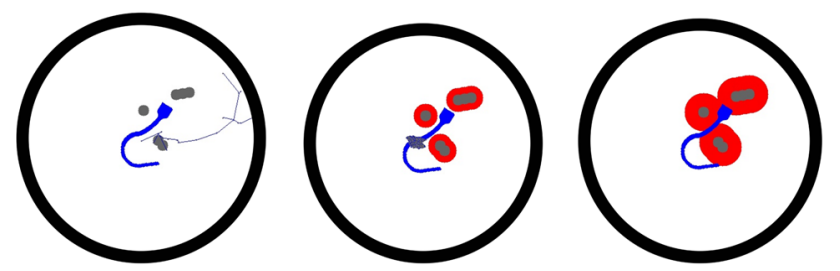

Fig. 5 From left to right: the object (hook) can escape only in the first case, and becomes completely caged when we enlarge the caging tools before a collision with the object occurs

size of the offset $0<\varepsilon_{\text {closed }}<\varepsilon_{c o l}$, such that the object is caged by the enlarged caging tools, but is not in collision. This is not always possible, as in certain situations the object may never become caged before colliding with enlarged caging tools. Figure 4 illustrates this situation.

Let us formally describe this situation. Let $\mathcal{C}_{\text {free }}^{\varepsilon}$ be the free space of the object induced by $\varepsilon$-offset of caging tools. As we increase the size of the offset, we get a nested family of spaces $\mathcal{C}_{\text {free }}^{\varepsilon_{\text {col }}} \subset \ldots \subset \mathcal{C}_{\text {free }}^{\varepsilon} \subset \ldots \subset \mathcal{C}_{\text {free }}^{0}$, where $\varepsilon_{\text {col }}$ is the smallest size of the offset causing a collision between the object and the enlarged caging tools. There are two possible scenarios: in the first one, there is a value $0<\varepsilon_{\text {closed }}<$ $\varepsilon_{c o l}$ such that when the offset size reaches it the object is caged by the enlarged caging tools. This situation is favorable for robotic manipulation settings, as in this case the object has some freedom to move within a partial cage, but cannot escape arbitrarily far as its mobility is limited by a narrow gate (see Fig. 5). ${ }^{3}$

We denote the set of all configurations falling into this category as the caging subset $\mathcal{C}_{\text {cage }}$. These configurations are promising partial cage candidates, and our primary interest is to identify these configurations. In the second scenario, for any $\varepsilon$ between 0 and $\varepsilon_{c o l}$, the object is not caged in the respective free space $\mathcal{C}_{\text {free }}^{\varepsilon}$, as shown in Fig. 4.

We define the notion of partial caging as follows:

Definition 3 Any configuration $c \in \mathcal{C}_{\text {cage }}$ of the object is called a partial cage of clearance $Q_{c l}(c)$.

\footnotetext{
${ }^{3}$ In Fig. 5 the enlarged caging tools (in red) cage the hook by trapping the larger base.
}

Note that the case where $\mathcal{E} \mathcal{P}\left(\mathcal{C}_{\text {cage }}, c\right)=\emptyset$ corresponds to the case of a complete (i.e., classical) cage. Thus, partial caging is a generalization of complete caging.

Based on this theoretical framework, we propose a partial caging evaluation process that consists of two stages. First, we determine whether a given configuration belongs to the caging subset $\mathcal{C}_{\text {cage }}$. If it does, we further evaluate its clearance with respect to our clearance measure $Q_{c l}$, where, intuitively, configurations with smaller clearance are considered more preferable for grasping and manipulation under uncertainty.

\section{Gate-based clearance estimation algorithm}

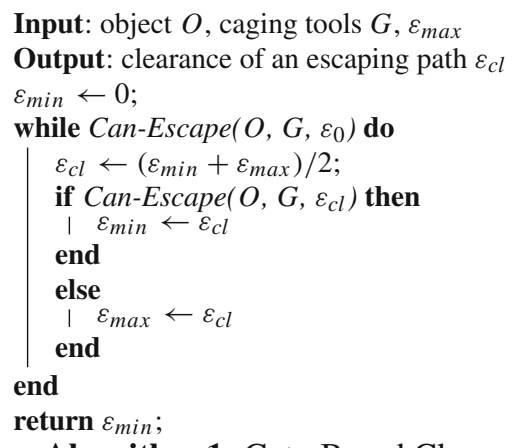

Algorithm 1: Gate-Based Clearance Estimation

In this section, we propose a possible approach to estimate $Q_{c l}(c)$-the Gate-Based Clearance Estimation Algorithm. Instead of finding a path with maximum clearance directly, we gradually inflate the caging tools by a distance offset until the object becomes completely caged. For this, we first approximate the object and the caging tools as union of discs, see Fig. 8. This makes enlarging the caging tools an easy task-we simply increase the radii of the discs in the caging tools' approximation by a given value. The procedure described in Algorithm 1 is then used to estimate $Q_{c l}(c)$.

We perform bisection search to find the offset value at which an object becomes completely caged. For this, we consider offset values between 0 and the radii of the workspace. We run RRT at every iteration of the bisection search in order to check whether a given value of the offset makes the object caged. In the experiments, we choose a threshold of 4 million iterations ${ }^{4}$ and assume that the object is fully caged if RRT

\footnotetext{
${ }^{4}$ Our experimental evaluation for our test dataset suggested that if after 4 million iterations RRT had not found an escaping path, then the object was caged with overwhelming likelihood. We thus considered RRT with this setting to provide a sufficiently good approximation for training the neural network.
} 
does not produce an escaping path at this offset value. Note that this procedure, due to the approximation with RRT up to a maximal number of iterations, does not guarantee that an object is fully caged; however, since no rigorous bound on the number of iterations made by RRT is known, we choose a threshold that performs well in practice since errors due to this RRT-based approximation become insignificant for sufficiently large maximal numbers of RRT sampling iterations. In Algorithm 1, Can-Escape $\left(O, G, \varepsilon_{c l}\right)$ returns True if the object can escape and is in a collision-free configuration.

\section{Grasping favorable configuration in $\mathcal{C}_{\text {cage }}$}

Depending on the size of the object with respect to the workspace, the bisection search performed in Algorithm 1 can be computationally expensive. Uniformly sampling caging tools placements from the entire workspace in order to find configurations in $\mathcal{C}_{\text {cage }}$ is also rather inefficient and the number of partial caging configurations of high quality can be low.

Furthermore, not all partial caging configurations defined by Definition 3 ( $c \in \mathcal{C}_{\text {cage }}$ ) are equally suitable for certain applications like grasping or pushing under uncertainty. Namely, we would like to place caging tools such that they are not too close and not too far away from the object.

To overcome these limitations, we define a region around the object called partial caging grasping band (Fig. 6 illustrates this concept):

Definition 4 Let $O$ be an object and assume the caging tools have a maximal width ${ }^{5} t_{d}$. Let $O_{\min }$ and $O_{\max }$ be objects where the composing disks are enlarged by $d i s_{\text {min }}=\frac{1}{2} c t_{d}$. $(1+\beta)$ and $d i s_{\max }=d i s_{\min }+\frac{1}{2} c t_{d} \cdot \gamma$ respectively.

We can then define the grasping band as follows:

$\mathcal{G B}=\left\{x \in \mathcal{C}_{\text {free }}:\left(x \in O_{\text {min }}\right) \oplus\left(x \in O_{\text {max }}\right)\right\}$,

Here, $\beta$ and $\gamma$ are parameters that capture the impreciseness of the system, such as vision and control uncertainties.

\section{Learning planar $Q_{c l}$}

As RRT is a non-deterministic algorithm, one would need to perform multiple runs in order to estimate $Q_{c l}$. In real-time applications, we would like the robot to be able to evaluate caging configurations within milliseconds. Thus, the main

\footnotetext{
5 The caging tools are composed of disks with $c t_{d}$ as diameter. As we only consider composed line configurations as caging tools the width never exceeds $c t_{d}$.
}

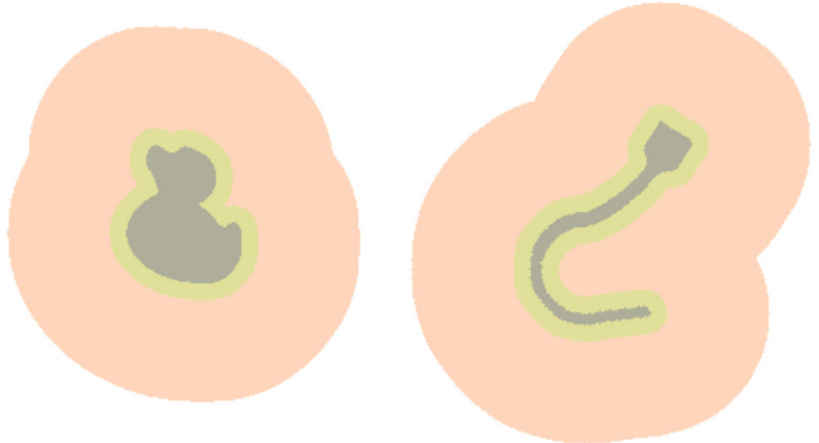

Fig. 6 An illustration of a grasping band for a duck and hook object. The object $O$ is in the center (gray) overlaid by $O_{\min }(O$ enlarged by $d i s_{\min }$, light green) overlaid by $O_{\max }\left(O\right.$ enlarged by $d i s_{\max }$, light orange). The grasping band $(\mathcal{G B})$ is the disjunctive union between $O_{\min }$ and $O_{\max }$ (Color figure online)

obstacle on the way towards using the partial caging evaluation function defined above in real time is the computation time needed to evaluate a single partial caging configuration.

Algorithm 1 requires several minutes to evaluate a single partial cage, while a neural network can potentially estimate a configuration in less than a second.

To address this limitation of Algorithm 1, we design and train two convolutional neural networks. The first, called CageMaskNN, acts as a binary classifier that identifies configurations that belong to $\mathcal{C}_{\text {cage }}$ following Def 3 . The second, architecturally identical network, called CageClearanceNN, approximates the caging evaluation function $Q_{c l}$ to estimate the quality of configurations. The network takes two images as input that correspond to the object and the caging tools. The two networks are separated to make training more efficient, as both can be trained independently. Operating both networks sequentially results in pipeline visualized in Fig. 1: first, we identify if a configuration is a partial cage, and if it is, we evaluate its quality.

Our goal is to estimate $Q_{c l}$ given $O \subset \mathbb{R}^{2}$-an object in a fixed position, and $G=\left\{g_{1}, g_{2}, . ., g_{n}\right\}$-a set of caging tools in a particular configuration. We assume that caging tools are normally disconnected, while objects always have a single connected component. In our current implementation, we consider $n \in\{3,4\}$, and multiple caging tool shapes.

While neural networks require a significant time to train (often multiple hours), evaluation of a single configuration is a simple forward pass through the network and its complexity is therefore not relying on the input size or data size but rather on the number of neurons in the network. In this work, our goal is to show that we can successfully train a neural network that can generalise to unseen input configurations and approximate the Algorithm 1 in milliseconds. 


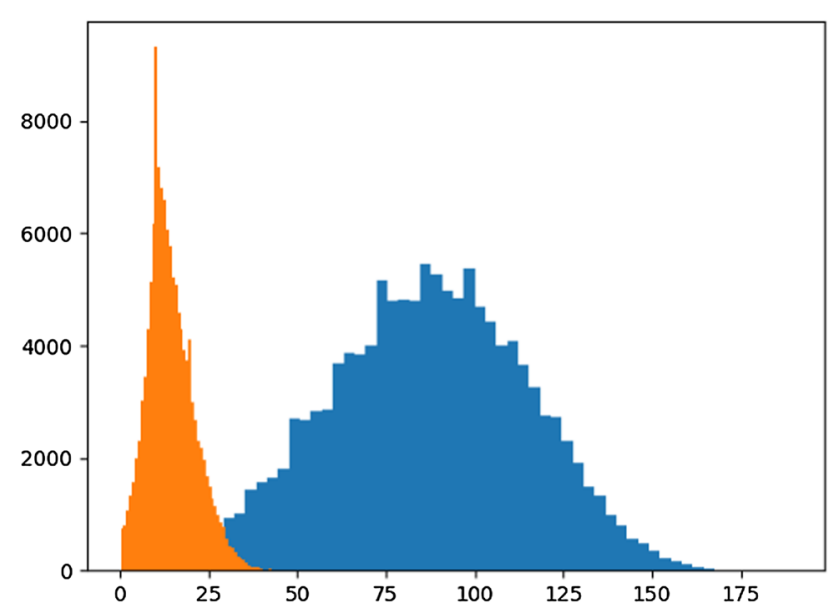

Fig. 7 Distribution of $Q_{c l}$ estimates for the $P C$-general (blue) and the $P C$-band (orange) datasets (Color figure online)

\subsection{Dataset generation}

We create a dataset of 3811 object models consisting of two-dimensional slices of objects' three-dimensional mesh representations created for the Dex-Net 2.0 framework (Mahler et al. 2017). We further approximate each model as a union of one hundred discs, to strike a balance between accuracy and computational speed. The approximation error is a ratio that captures how well the approximation $\left(A_{\text {app }}\right)$ represents the original object ( $A_{\text {org }}$, and is calculated as follows: $a_{e}=\frac{A_{\text {org }}-A_{\text {app }}}{A_{\text {org }}}$. Given the set of objects, two partial caging datasets are generated. The first dataset, called $P C$-general, consists of 3811 objects, 124435 partial caging configurations (belonging to $\mathcal{C}_{\text {cage }}$ ), and 18935565 configurations that do not belong to $\mathcal{C}_{\text {cage }}$.

One of the limitations of the $P C$-general dataset is that it contains relatively few partial caging configurations of high quality. To address this limitation, generate a second partial caging dataset called PC-band where caging tools placements are only located inside the grasping bands of objects, as this strategy increases the chance that the configuration will be a partial cage of low $Q_{c l}$ as well as the likelihood of a configuration belonging to $\mathcal{C}_{\text {cage }}$.

The $P C$-band dataset consists of 772 object with 3,785,591 configurations of caging tools, 127,733 of which do belong to the partial caging subset $\mathcal{C}_{\text {cage }}$. We set $\beta$ to the approximation error $a_{e}$ for each object and $\gamma=6$ to define the grasping band.

All configurations are evaluated with $Q_{c l}$ (see Algorithm 1). The distribution of partial cages can be seen in Fig. 7.

Examples of configurations for both datasets can be seen in Fig. 8. The disk approximation of the object is shown in blue, while the original object is depicted in red. $P C$-general contains configurations placed in the entire workspace while

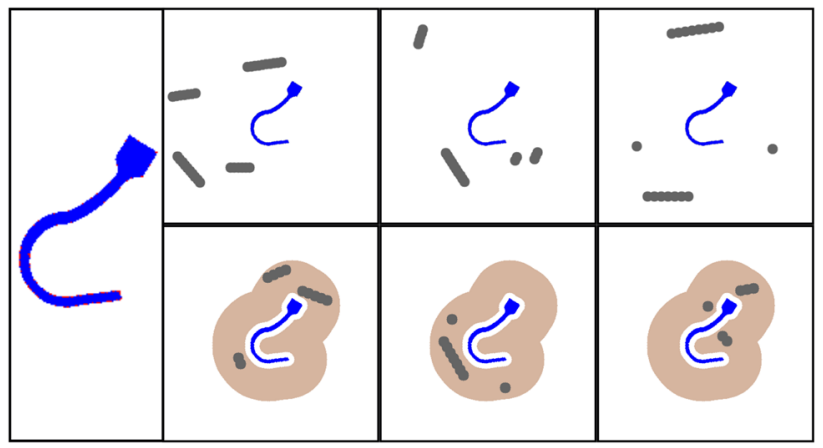

Fig. 8 Left: original representations of a hook objects (red) and in blue their approximation by a union of discs of various sizes closely matching the polygonal shape $\left(a_{e}=0.051\right)$; second and third column: configurations that do not belong to $\mathcal{C}_{\text {cage }}$; last column: a partial caging configuration $\left(c \in \mathcal{C}_{\text {cage }}\right)$. The top row is from $P C$-general, the bottom from $P C$-band (Color figure online)

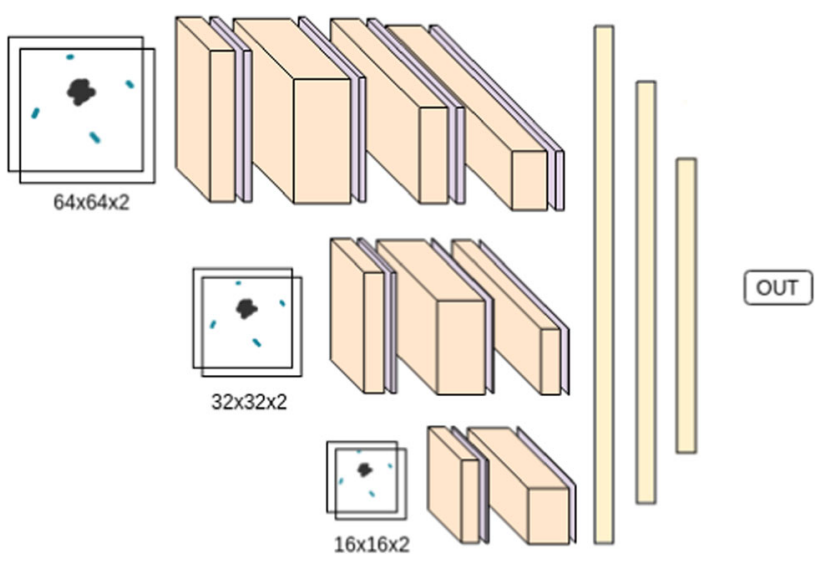

Fig. 9 As caging depends on global geometric properties of objects, a $\mathrm{CNN}$ architecture with multi-resolution input was designed to capture these features efficiently

$P C$-band is limited to configuration sampled inside the grasping band.

\subsection{Architecture of convolutional neural networks}

We propose a multi-resolution architecture that takes the input image as $64 \times 64 \times 2,32 \times 32 \times 2$, and $16 \times 16 \times 2$ tensors. This architecture is inspired by inception blocks (Szegedy et al. 2014). The idea is that the global geometric structure can be best captured with different image sizes, such that the three different branches can handle scale-sensitive features. The network CageMaskNN determines whether a certain configuration belongs to $\mathcal{C}_{\text {cage }}$, while CageClearanceNN predicts the clearance $Q_{c l}$ value for a given input configuration.

The architecture of the networks is shown in Fig. 9. Both networks take an image of an object and caging tools on a uniform background position and orientation belonging to the same coordinate frame constituting a two-channel image $(64 \times 64 \times 2)$ as input. CageMaskNN performs binary classifi- 
cation of configurations by returning 0 in case a configuration belongs to $\mathcal{C}_{\text {cage }}$, and 1 otherwise. CageClearanceNN uses clearance $Q_{c l}$ values as labels and outputs a real valuethe predicted clearance of a partial cage. The networks are trained using the Tensorflow (Abadi et al. 2016) implementation of the Adam algorithm (Kinga and Adam 2015). The loss is defined as the mean-squared-error (MSE) between the prediction and the true label. The batch size was chosen to be 100 in order to compromise between learning speed and gradient decent accuracy. The networks were trained on both of our datasets-PC-general and PC-band.

\section{Training and evaluation of the networks}

In this section we describe how we train and evaluate the two networks and perform an ablation study of the architecture. In detail, for CageMaskNN, we investigate to what extent the training data should consist of samples belonging to $\mathcal{C}_{\text {cage }}$ and evaluate the performance of the best such composition against a simpler network architecture. Following that, we investigate how the number of different objects as well as the choice of dataset influences the performance of CageMaskNN.

For CageClearanceNN, we also perform an analysis of the effect of the the number of objects in the training data and to what extent the choice of dataset influences the performance and compare it to a simpler architecture. As a final investigation, we investigate the error for specific $Q_{c l}$ intervals.

Note that the training data is composed of samples where the ground truth of the configuration was obtained using Algorithm 1. A main goal of the presented evaluation is hence to investigate how well the proposed networks are able to generalise to examples that were not included in the training data (unseen test data). High such generalization performance, is a key indicator for the potential application of the proposed fast neural network based approach (execution in milliseconds) instead of the computationally expensive underlying Algorithm 1 (execution in minutes) that was used to generate the training data.

Single-res Architecture In order to perform an ablation of the previous discussed multi-resolution architecture we compare the performance so a architecture that has only a single resolution as input. The Single-res Arch. Takes only the $64 \times 64 \times 2$ as input and is missing the other heads completely. In this way we want to see if our assumption that different sized inputs are beneficial to the networks performance.

\subsection{CageMaskNN-\% of $\mathcal{C}_{\text {cage }}$ and ablation}

We generate 4 datasets containing 5\%,10\%,15\%, and $20 \%$ caging configurations in $\mathcal{C}_{\text {cage }}$ respectively from $P C$-general. This is achieved by oversampling as well as by performing
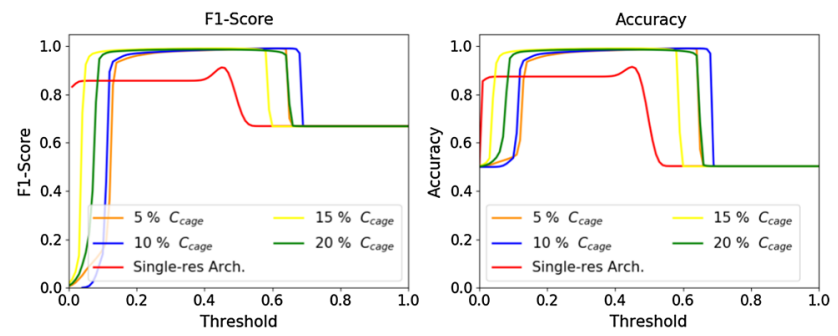

Fig. 10 F1-score and accuracy of the network depending on different thresholds

rotational augmentation with 90,180 and 270 degrees of the existing caging configurations. The Single-res Arch. is trained with $10 \%$ caging configurations in $\mathcal{C}_{\text {cage }}$ for comparison.

The evaluation is performed on a test set consisting of 50\% caging examples from $\mathcal{C}_{\text {cage }}$. In Fig. 10, we show the F1-curve and Accuracy-curve. All five versions of the network where trained with 3048 objects with 2000 configuration each, using a batch size of 100 and 250000 iterations. To avoid overfitting, a validation set of 381 objects is evaluated after every $100^{\text {th }}$ iteration. The final scoring is done on a test set consisting of 381 previously unseen objects. The mean squared error (MSE) on the unseen test set was $0.0758,0.0634,0.0973$ and 0.072 for the $5 \%, 10 \%, 15 \%$ and $20 \%$ version respectively, indicating that CageMaskNN is able to generalize to novel objects and configurations from our test set. The MSE for the single resolution network was 0.155 showing the significant gain obtained by utilizing the multi-resolution branches.

We observe that the network that was trained on the dataset where $10 \%$ of the configurations are partial cages performs slightly better than the other versions. Note however that only the one that was trained with $5 \%$ of partial cages performs significantly worse. All versions of the multiresolution architecture outperform the Single-res Arch, which justifies our architecture design.

\subsection{CageMaskNN—number of objects and datasets}

We investigate how the performance of the networks depends on the size of the training data and how the two training datasets, $P C$-general and $P C$-band, affect the performance of the networks. Table 1 shows the area under ROC curve (AUC) andthe average precision (AP) for CageMaskNN for training set composed of 1, 10, 100, and 1000 objects from the dataset $P C$-general, as well as 1, 10,100, and 617 objects from $P C$-band. We observe that having more objects in the training set results in better performance. We note that the network trained on $P C$-general slightly outperforms the one trained on $P C$-band.

Figure 11 demonstrates how the performance of the networks increases with the number of objects in the training 
Table 1 The area under ROC curveThe area under ROC curve (AUC) and the average precision (AP) for different training set constitutions, evaluated on the test set with $50 \%$ of partial cage configurations

\begin{tabular}{llllll}
\hline Training set & \multicolumn{2}{l}{ pc-general } & & \multicolumn{2}{l}{ pc-band } \\
\cline { 2 - 3 } & AUC & AP & & AUC & AP \\
\hline 1 object & 0.92 & 0.88 & & 0.88 & 0.83 \\
10 objects & 0.91 & 0.88 & & 0.88 & 0.84 \\
100 objects & 0.97 & 0.92 & & 0.92 & 0.89 \\
1000|| 617 objects & 1.00 & 1.00 & & 1.00 & 0.96 \\
\hline
\end{tabular}

In all training sets $10 \%$ of configurations belong to $C_{\text {cage }}$. We observe that $P C$-general has a slightly better performance than $P C$-band
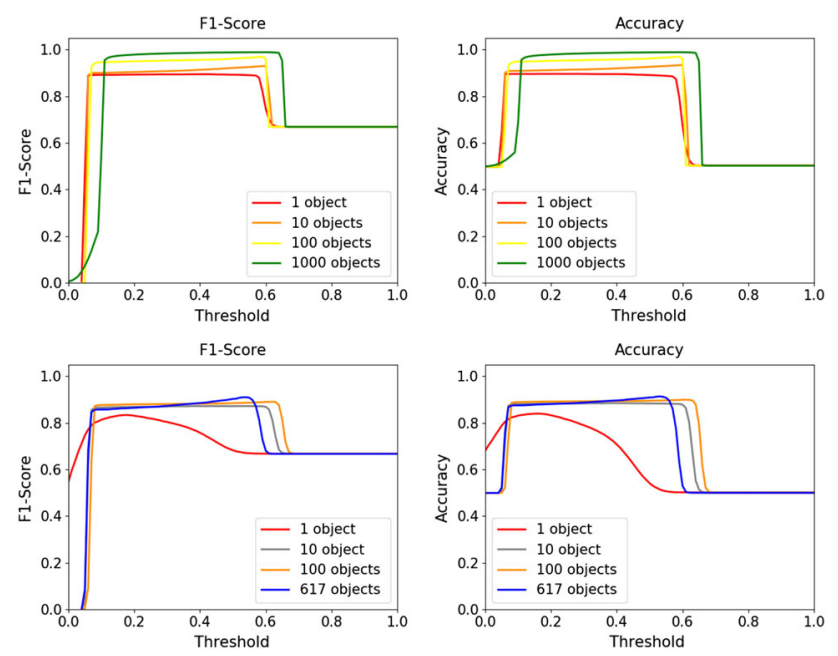

Fig. 11 F1-score and accuracy of the network trained with 1, 10, 100, and 1000|| 617 objects,for $P C$-general (top row) and $P C$-band (bottom row) respectively on a test set with $50 \% C_{\text {cage }}$ configuration

dataset by showing the F1-score as well as the accuracy for both datasets. We observe that the network, independently of the training dataset, demonstrates acceptable performance even with a modest numbers of objects in the training dataset. One key factor here is the validation set which decreases the generalisation error by choosing the best performance during the entire training run, thus reducing the risk of overfitting. Similarly to the previous results, $P C$-general slightly outperforms $P C$-band.

\subsection{CageClearanceNN - Number of Objects and Ablation}

The purpose of CageClearanceNN is to predict the value of the clearance measure $Q_{c l}$ given a partial caging configuration. We trained CageClearanceNN on 1, 10, 100,1000 and 3048 objects from $P C$-general as well as a single resolution variant with the same training sets. Additionally, we trained another instance of CageClearanceNN with 1, 10, 100, and 617 objects from PC-band, and the correspond-
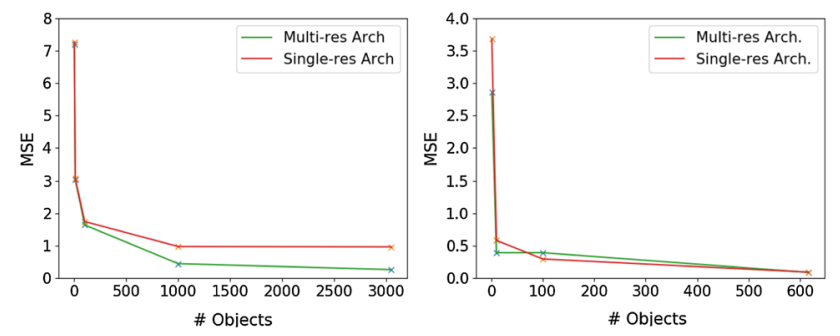

Fig. 12 Left: MSE of CageClearanceNN trained on PC-general with different numbers of objects and a single-resolution architecture; right: MSE of the single-resolution architecture trained on $P C$-band with different numbers of objects

ing single-resolution architecture version for each number of objects. The label is scaled with a factor of 0.1 , as we found that the networks performance improves for smaller training input values. The left-hand side of Fig. 12 shows a rapid decrease of MSE as we increase the number of training data objects to 1000 , and a slight performance increase between 1000 and 3048 training objects for the $P C$-general dataset. We can also see that employing the multi-resolution architecture only leads to significant performance increase when going up to 1000 objects and more. The right-hand side of Fig. 12 presents the analogous plot for the network trained on PC-band. We observe the same rapid decrease of MSE as we include more objects in the training set. Note that the different number of parameter plays a role as well in the performance difference. Since our current dataset is limited to 617 training examples of object shapes, we do not observe the benefits of the multi-resolution architecture. Note that the difference in absolute MSE stems from the different distributions of the two datasets (as can be seen in Fig. 7). This indicates that further increases in performance can be gained by having more training objects. Increasing the performance for more than 3000 objects may however require a significant upscaling of the training dataset.

\subsection{CageClearanceNN - Error for specific $Q_{c l}$}

We investigated the MSE for specific $Q_{c l}$ value intervals. Figure 13 shows the MSE on the test set with respect to the $Q_{c l}$ values (as before, scaled by 0.1). Unsurprisingly, we observe that the network, trained on $P C$-general, that was trained only on one object, does not generalise over the entire clearance/label spectrum. As we increase the number of objects, the performance of the network increases. The number of outliers with large errors decreases significantly when the network is trained on 1000 objects. On the right side, we can see the MSE for the final CageClearanceNN network trained on $P C$-general. We observe that low values of $Q_{c l}$ are associated to higher error values. Analysing this behavior on CageClearanceNN trained on PC-band demonstrates a very similar behavior and is therefore omitted. 


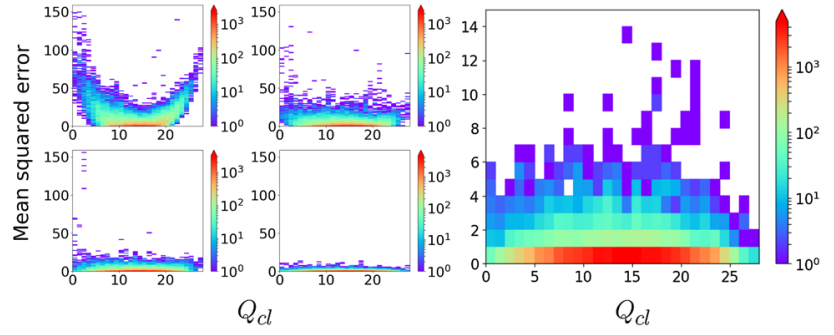

Fig. 13 MSE for each test case sorted for labels. Left: shows performance of 1,10,100, 1000 objects (top left, top right, bottom left, bottom right). Right: shows MSE of entire test set for the final CageClearanceNN. Note that the figure on the right is zoomed in as errors are significantly smaller (see the left $y$-axis of that figure)

\section{Planar Caging Pipeline Evaluation}

\subsection{Last caging tool placement}

In this experiment, we consider the scenario where $n-1$ out of $n$ caging tools are already placed in fixed locations, and our framework is used to evaluate a set of possible placements for the last tool to acquire a partial cage. We represent possible placements as cells of a two-dimensional grid and assume that the orientation of the caging tool is fixed. Figure 14 illustrates this approach.

We use the pipeline trained with $P C$-general as it covers the entire workspace.

In the example $a$, we can see that placing the caging tool closer to the object results in better partial caging configurations. This result is consistent with our definition of the partial caging quality measure. We note furthermore, that CageMaskNN obtains an approximately correct region-mask of partial caging configurations for this novel object. Example $b$ demonstrates the same object with elongated caging tools. Observe that this results in a larger region for possible placement of the additional tool. Example $c$ depicts the same object but the fixed disc-shaped caging tool has been removed and we are considering three instead of four total caging tools. This decreases the number of possible successful placements for the additional caging tool. We can see that our framework determines the successful region correctly, but is more conservative than the ground truth. In the example $d$, we consider an object with two large concavities and three caging tools. We observe that CageMaskNN identifies the region for $C_{\text {cage }}$ correctly and preserves its connectivity. Similarly to the previous experiments, we can also observe that the most promising placements (in blue) are located closer to the object.

\subsection{Evaluating $Q_{c l}$ along a trajectory}

We now consider a use case of $Q_{c l}$ along a caging tool trajectory during manipulation enabled by the fact that the
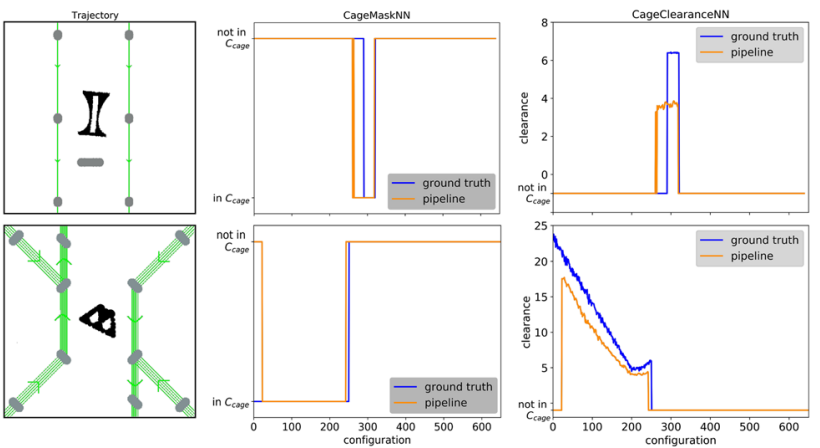

Fig. 14 Here, we depict the results of four different experiments. The green region indicates configuration where the additional caging tool completes the configuration in such a way that the resulting configuration is a partial cage. The small squares in the ground truth figures depict the caging tools that are being placed (for simplicity the orientations are fixed). We plot the output for each configuration directly and visualize the result as a heatmap diagram (blue for partial caging configurations, white otherwise). The best placements according to CageClearanceNN are depicted in dark blue, and the worst ones in yellow. The results are normalized between 0 and 1 . Grey area corresponds to the placements that would result in a collision (Color figure online)

evaluation of a single caging configuration using CageMaskNN and CageClearanceNN takes less than $6 \mathrm{~ms}$ on a GeForce GTX 1080 GPU.

The results for two simulated sample trajectories are depicted in Fig. 15. In the first row, we consider a trajectory of two parallel caging tools, while in the trajectory displayed in the bottom row, we consider the movement of 4 caging tools: caging tool 1 moves from the top left diagonally downwards and then straight up, caging tool 2 enters from the bottom left and then exits towards top, caging tool 3 enters from the top right and then moves downwards, while caging tool 4 enters from the bottom right and then moves downwards.

The identification of partial caging configurations by CageMaskNN is rather stable as we move the caging tool along the reference trajectories, but occurs at a slight offset from the ground truth. The offset in CageClearanceNN is larger but consistent, which can be explained by the fact that similar objects seen during training had a lower clearance as the novel hourglass shaped object. In the second example, the clearance of the partial cage decreases continuously as the caging tools get closer to the object. Predicted clearance values from CageClearanceNN display little noise and low absolute error relative to the ground truth. Note that a value of -1 in the quality plots refers to configurations identified as not being in $\mathcal{C}_{\text {cage }}$ by CageMaskNN.

\subsection{Experimental evaluation of $Q_{c l}$}

In this section, we experimentally evaluate our partial caging quality measure $Q_{c l}$ by simulating random shaking of the caging tools and measuring the needed time for the object to 
(a)

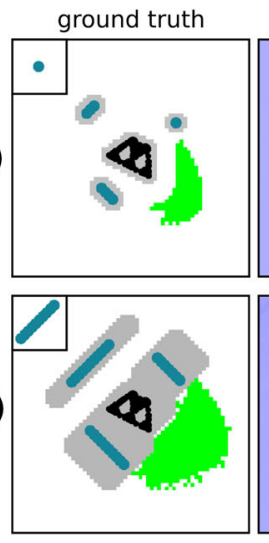

CageMaskNN
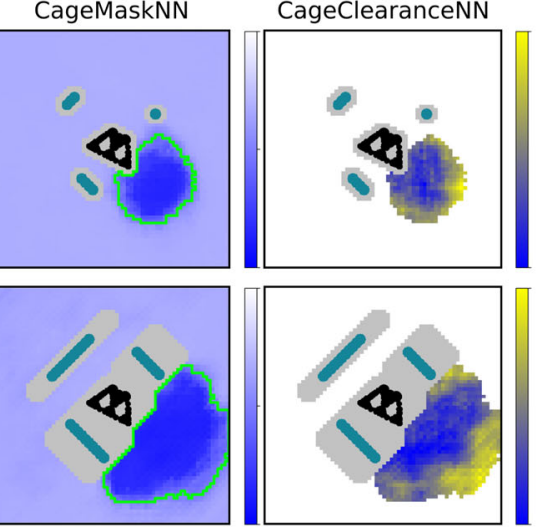

Fig. 15 Evaluation of the pipeline along two trajectories. The trajectory (left, green) is evaluated with CageMaskNN (middle) and CageClearanceNN (right), which evaluates $Q_{c l}$ for those configurations where

escape. Intuitively, the escape time should be inversely proportional to the estimated $Q_{c l}$; this would indicate that it is difficult to escape the partial cage. A similar approach to partial caging evaluation has been proposed in Makapunyo et al. (2012). Where the escape time was computed using probabilistic motion planning methods like RRT, RRT*, PRM, SBL as well as a random planner was measured.

\subsubsection{Random partial caging trajectories}

We apply a simple random walk $X_{n}$ as a sequence of independent random variables $S_{1}, S_{2}, \ldots, S_{n}$ where each $S$ is is randomly chosen from the set $\{(1,0),(0,1),(1,1),(-1,0),(0$, $-1),(-1,-1)\}$ with equal probability.

$\left.X_{n}=X_{0}+S_{1}+S_{2}+\cdots+S_{n}\right)$,

where $X_{0}$ is the start position of the caging tools. and a stride factor $\alpha$ determines at what time the next step of the random walk is performed.

In this experiment, unlike in the rest of the paper, caging tools are moving along randomly generated trajectories. We assume that the object escapes a partial cage when it is located outside of the convex hull of the caging tools. If the object does not escape within $t_{\max }$ seconds, the simulation is stopped. The simulation is performed with the software pymunk that is build on the physic engine Chipmunk 2D (Lembcke 2013). We set the stride factor $\alpha=0.05 s$ so that a random step $S$ of the random walk $X_{n}$ is applied to the caging tool every 0.05 seconds. As pymunk also facilitates object interactions, the caging tool can push the object around as well as drag it with them. Figure 16 illustrates this process.

The experiment was performed on 5 different objects, depending on the object we used between 437 and 1311 (c)

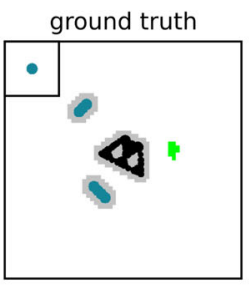

CageMaskNN

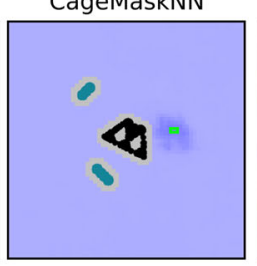

CageClearanceNN

(d)
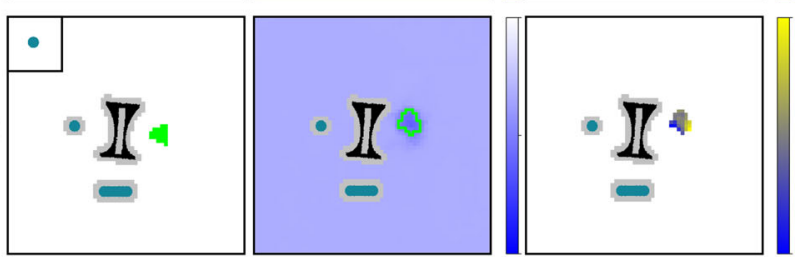

CageMaskNN returns 0. The predictions by the networks are displayed in orange while ground truth is shown in blue (Color figure online)
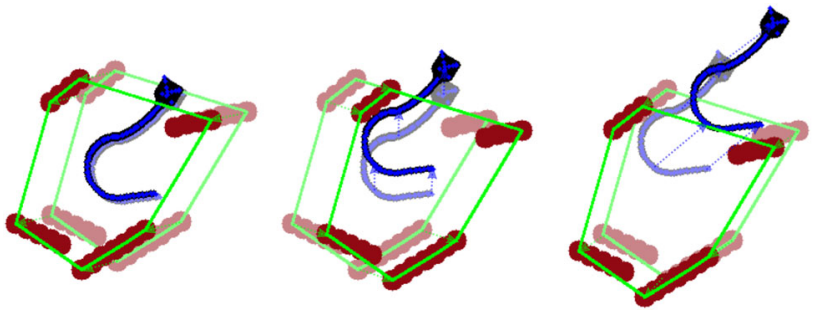

Fig. 16 Random trajectory for caging tools. Left: time $t=$ $0 s$ (transparent) to $t=0.83 \mathrm{~s}$ (not escaped), middle: $t=$ $0.83 \mathrm{~s}$ (transparent) to $t=1.67 \mathrm{~s}$ (not escaped), right: time $t=1.67 \mathrm{~s}$ (transparent) to $t=2.47 \mathrm{~s}$ (escaped). Note that the caging tools do not necessarily run in a straight line but rather follow the randomly generated trajectory with a new step every $0.05 \mathrm{~s}$. As a simple physics simulator is used, the caging tools can also induce movement of the object by colliding with it

caging tool configurations. For each of them the escape time was estimated as described above. As it is not deterministic, we performed 100 trials for each configuration and computed the mean value. The mean escape time of 100 trials was normalized such that the values range between 0 and 1 . Furthermore, for each configuration we computed $Q_{c l}$ and the Pearson correlation coefficient. ${ }^{6}$ Fig. 17 illustrates the results.

Our results show that the longer it takes for the object to escape the partial cage, the higher the variance of the escape time is. This indicates that a partial cage quality estimate based on the average escape time would require a high number of trials, making the method inefficient.

Furthermore, we demonstrate that our clearance-based partial caging quality measure shows a trend with the aver-

\footnotetext{
6 The Pearson correlation coefficient measures the linear correlation between the escape time from random shaking and the defined clearance measure $Q_{c l}$.
} 

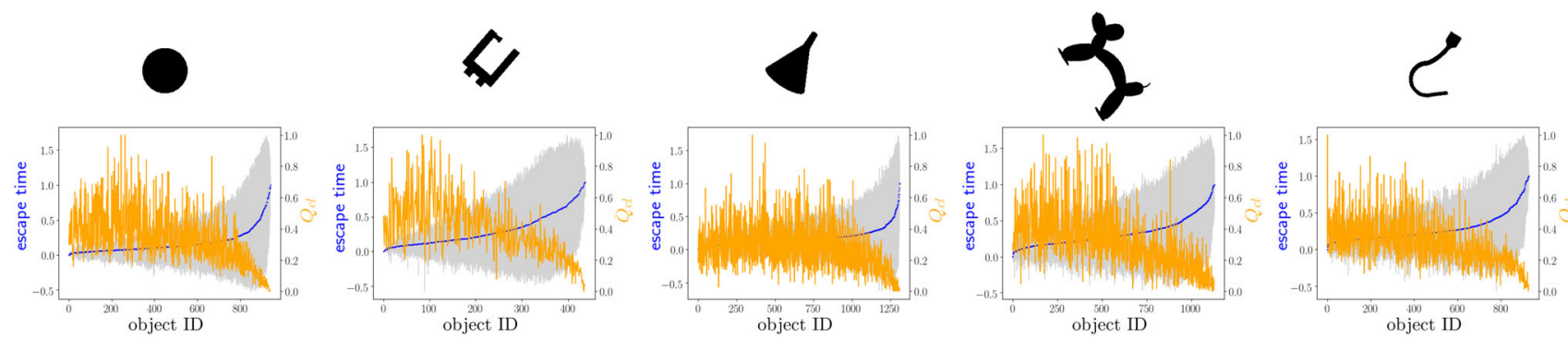

Fig. 17 Correlation between escape time from random shaking and $Q_{c l}$. Top row shows evaluated objects (disk, clench, cone, balloon animal, and hook, on the bottom row the partial cages are sorted according to respective average escape time, and plot the average escape time (in blue), its variance (in gray), and $Q_{c l}$ (in orange). Pearson correlation coefficient of the escape time and $Q_{c l}$ (from left to right) are: -0.608 , $-0.462,-0.666,-0.566,-0.599$ (Color figure online)
Table 2 Average results for 500 novel objects cage acquisition using different distance metrics to find similar objects in $P C$-band, and applied cages from retrieved objects to novel objects

\begin{tabular}{|c|c|c|c|c|c|c|}
\hline & \multicolumn{2}{|c|}{ Collision-free } & \multicolumn{2}{|c|}{$\frac{\text { Cage evaluation }}{\text { top } 1}$} & & \\
\hline & Top $1(\%)$ & Top $5(\%)$ & $\in \mathcal{C}_{\text {cage }}(\%)$ & $Q_{c l}$ & $\in \mathcal{C}_{\text {cage }}(\%)$ & $Q_{c l}$ \\
\hline VAE & 90.9 & 86.6 & 53.2 & 5.05 & 48.4 & 5.92 \\
\hline Hausdorff & 75.5 & 75.2 & 33.6 & 5.21 & 32.2 & 5.83 \\
\hline Hamming & 74.4 & 73.9 & 35.4 & 3.87 & 34.3 & 4.51 \\
\hline Random & 61.6 & 62.3 & 27.0 & 13.31 & 25.4 & 14.12 \\
\hline
\end{tabular}

age escape time for strong partial cages, which suggests the usefulness of the proposed measure.

\subsection{Different metrics in the space of shapes for partial caging}

A natural extension of our partial caging evaluation framework is partial cage acquisition: given a previously unseen object, we would like to be able to quickly synthesise partial cages of sufficient quality. In this section, we make the first step in this direction, and propose the following procedure: given a novel object, we find similar objects from the training set of the $P C$-band, and consider those partial caging configurations that worked well for these similar objects.

The key question here is how to define a distance function for the space of objects that would capture the most relevant shape features for partial caging. In this experiment, we investigate three different shape distance functions: Hausdorff distance, Hamming distance, and Euclidean distance in the latent space of a variational autoencoder, trained on the set of objects used in this work. Variational autoencoders (VAEs) are able to encode high-dimensional input data into a lower-dimensional latent space while training in an unsupervised manner. In contrast to a standard encoder/decoder setup, which returns a single point, a variational autoencoder returns a distribution over the latent space, using the $K L$-cost term as regularisation.
We evaluate different distance functions with respect to the quality of the resulting partial cages. Given a novel object, we calculate the distance to each known object in the dataset according to the three distance functions under consideration, and for each of them we select five closest objects. When comparing the objects, orientation is an important factor. We compare 360 rotated versions of the novel object with the known objects from the dataset and pick the one closest following the chosen metric.

\subsubsection{VAE-based representation}

For our experiment, we train a VAE based on the ResNet architecture with skip connections with six blocks (Dai and Wipf 2019) for the encoder and the decoder. The imput images have resolution $256 \times 256$. We use a latent space with 128 dimensions, dropout of 0.2 and a fully connected layer of 1024 nodes. The VAE loss was defined as follows:

$$
\mathcal{L}_{\text {vae }}(x)=E_{z \sim q(z \mid x)}[\log p(x \mid z)]+\beta \cdot D_{K L}(q(z \mid x) \| p(z))
$$

The first term achives reconstruction, while the second term tries to disentegel the destinct features. $z$ denotes latent variable, $p(z)$ the prior distribution, and $q(z \mid x)$ the approximate posterior distribution. Note that the Bernoulli distribution was used for $p(x \mid z)$, as the images are of a binary nature.

The batch size was set to 32 . As the sizes of the objects vary significantly, we invert half of the images randomly when 
(a)

(b)

\begin{tabular}{|c|c|c|c|c|c|}
\hline \multirow{2}{*}{$\begin{array}{c}\text { query } \\
\boldsymbol{9}\end{array}$} & \multicolumn{5}{|c|}{ VAE } \\
\hline & 0 & 0 & 0 & & \\
\hline \multirow{3}{*}{ P } & & & usc & & \\
\hline & \} & 0 & 0 & B & $\diamond$ \\
\hline & \multicolumn{5}{|c|}{ Hamming } \\
\hline P & & & & & \\
\hline
\end{tabular}

(c)

\begin{tabular}{|c|c|c|c|c|c|}
\hline \multirow[b]{2}{*}{ P } & \multicolumn{5}{|c|}{ Random } \\
\hline & $\infty$ & 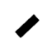 & - & 1 & 1 \\
\hline query & & & VAE & & \\
\hline$\succ$ & $\nabla$ & $\sigma_{3}$ & - & - & - \\
\hline
\end{tabular}

\begin{tabular}{|c|c|c|c|c|c|}
\hline \multirow[b]{2}{*}{$\longrightarrow$} & \multicolumn{5}{|c|}{ Hausdorff } \\
\hline & I & - & $/$ & - & r \\
\hline & \multicolumn{5}{|c|}{ Hamming } \\
\hline$\longrightarrow$ & $\gamma$ & I & 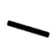 & 1 & 1 \\
\hline
\end{tabular}

\begin{tabular}{|c|c|c|c|c|c|}
\hline query & ᄀ & 8 & VAE & 3 & $\leftarrow$ \\
\hline \multirow[t]{2}{*}{ c } & 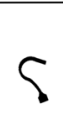 & $\checkmark$ & iusdo & $\checkmark$ & 9 \\
\hline & \multicolumn{5}{|c|}{ Hamming } \\
\hline \multirow[t]{2}{*}{$\zeta$} & حم & $i$ & - & d & 0 \\
\hline & \multicolumn{5}{|c|}{ Random } \\
\hline$c$ & 8 & هt & 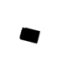 & 4 & $\checkmark$ \\
\hline
\end{tabular}

(a)

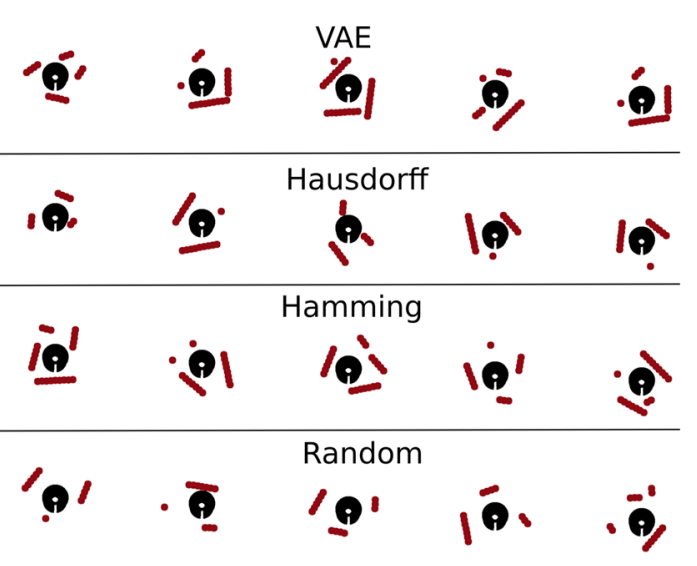

(b)

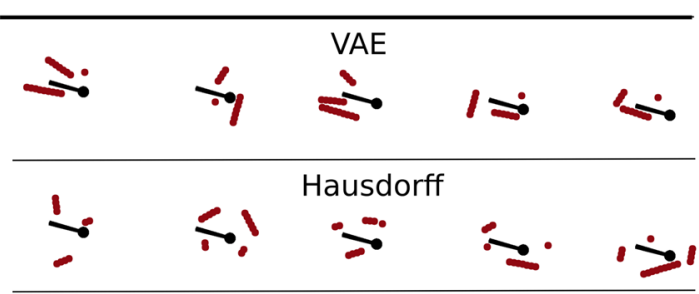

(c)
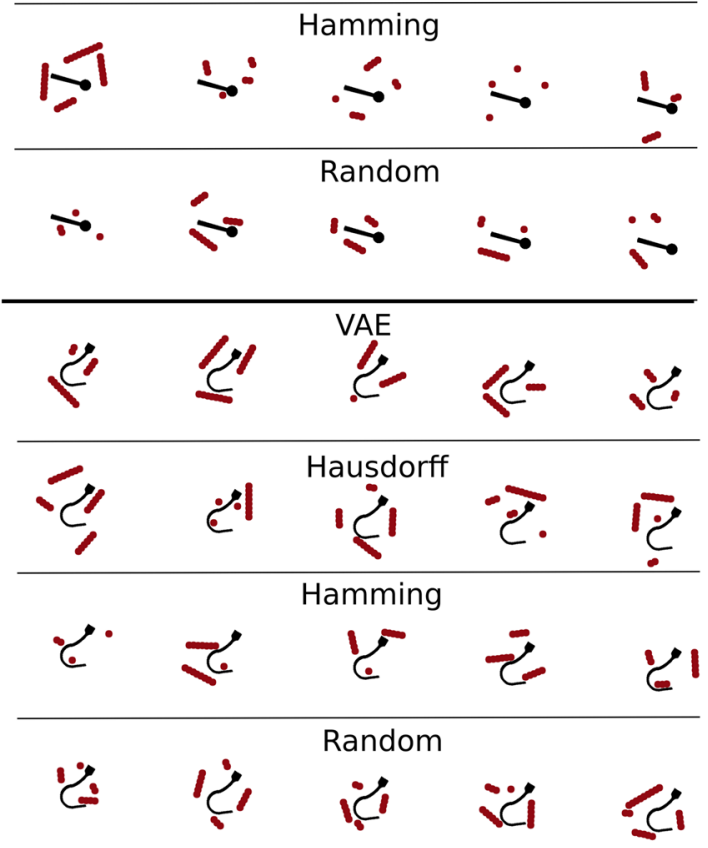

Fig. 18 On the left-hand side, we consider 3 different query objects washer (a), pin (b) and hook (c), and for each distance function visualize respective 5 closest objects from the training dataset; on the right-hand side, for each of the query object (a)-c)) and each distance function, we visualize the acquired partial caging configurations

our case). As the objects are represented with disks we use the set of $\mathrm{x}$ and $\mathrm{y}$ points to represent the object. This is a simplification of the object as the radius of the circles is not considered. The general Hausdorff distance can be computed

\subsubsection{Hausdorff distance}

The Hausdorff distance is a well known measure for the distance between two sets of points in a metric space $\left(\mathbb{R}^{2}\right.$ for 
with Taha and Hanbury (2015):

$d_{H}(X, Y)=\max \left\{\sup _{x \in X} \inf _{y \in Y} \mathrm{~d}(x, y), \sup _{y \in Y} \inf _{x \in X} \mathrm{~d}(x, y)\right\}$

\subsubsection{Hamming Distance}

The Hamming distance (Hamming April 1950) is defined as the difference of two binary data strings calculated using the XOR operation. It captures the exact difference between the two images we want to match, as it calculates how many pixel are different. We pre-process the images by subtracting the mean and reshaping the images to a $1 \mathrm{D}$ string.

\subsection{Performance}

We compare the performance of the three different similarity measures, as well as a random selection baseline, on 500 novel object. The percentage of collision-free caging tools placements, as well as the average clearance score is shown in Table 2. We report the average percentage of collision-free caging tool placements taken from the $P C$-band of partial cages for top 1 and top 5 closest objects.

Furthermore, we evaluate the collision-free configurations using Algorithm 1 to provide $Q_{c l}$ values as well as check if the configuration still belongs to $\mathcal{C}_{\text {cage }}$. In the Table 2 , the top 1 column under cage evaluation shows the percentage of configurations that belong to $\mathcal{C}_{\text {cage }}$. To the right is the average $Q_{c l}$ for the most promising cage from the closest object. The top 25 column shows the same results for the five most promising cages for each of the five closest objects. Examples for three novel objects and the closest retrieved objects are shown in Fig. 18. In the left column, the closest objects with respect to the chosen metric are shown given the novel query object. The right column shows the acquired cages, transferred from the closest known objects. Note that a collision free configuration does not necessarily have to belong to $\mathcal{C}_{\text {cage }}$.

For the VAE-model, it takes approximately 5 milliseconds to generate the latent representation, any subsequent distance query can then be performed in 0.005 milliseconds. The Hausdorff distance requires 0.5 milliseconds to compute, while the Hamming distance takes 1.7 milliseconds per distance calculation. $^{7}$

Our experiments show that, while the VAE-induced similarity measure performs best in terms of finding collision-free caging tools placements, Hamming distance significantly outperforms it in terms of the quality of acquired partial cages. We did not observe a significant difference between Hausdorff distance and the VAE-induced distance. While

\footnotetext{
7 The time was measured on a Intel(R) Core(TM) i7-7700HQ CPU @ $2.80 \mathrm{GHz}$.
}

(a)
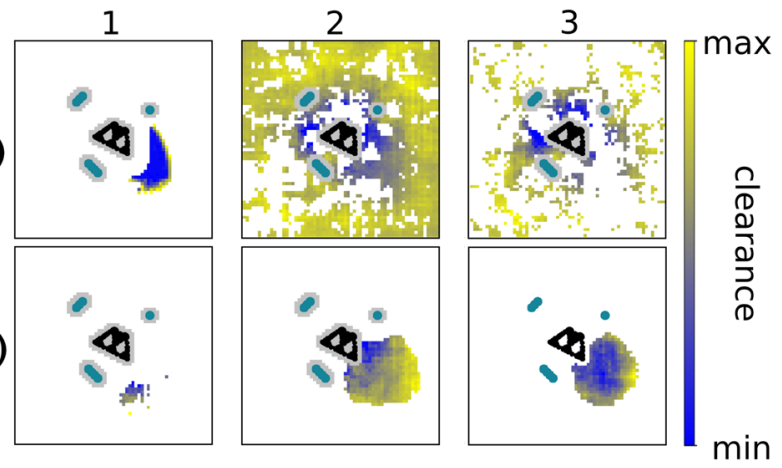

Fig. 19 Performance of CageMaskNN and CageClearanceNN given different numbers of training objects and evaluated on a single novel object. The top left (a1) displays the ground truth mask and clearance values for a fourth missing disc-shaped caging tool, a2: only 1 object is used for training, a3:10 objects are used for training, b1: 100 objects, b2: 1000 objects, b3: all 3048 objects are used for training. Note that the threshold had to be adjusted to 0.6 for the single object (a2) and 0.61 for the 10 object case (a3) to yield any discernible mask results at all

\begin{tabular}{|c|c|c|c|c|c|c|}
\hline query & & VAE & & & $\mathrm{mmin}$ & \\
\hline c & 1 & 3 & 日 & $\partial$ & $i$ & - \\
\hline$c$ & 1 & 3 & 日 & 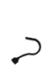 & $i$ & - \\
\hline$e$ & 1 & 3 & 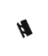 & 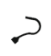 & $i$ & - \\
\hline c & Q & 9 & 2 & حر. & $i$ & $\cdot$ \\
\hline ¿ & Q & $\Leftrightarrow$ & $\theta$ & ح & $i$ & $\sim$ \\
\hline$\varepsilon$ & 1 & 3 & 日 & $\partial$ & $i$ & - \\
\hline$c$ & 1 & $M$ & 3 & $\nu$ & 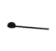 & $i$ \\
\hline$c$ & ? & N & 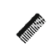 & $=$ & $\mathbb{d}$ & 0 \\
\hline$\ell$ & W & 9 & 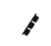 & 0 & I & 0 \\
\hline
\end{tabular}

Fig. 20 Top three retrieval results for query images with different levels of disturbance for the VAE-induced and Hamming metric. $a$ results without disturbance, $b$ show retrieval for different level of salt and pepper noise, $c$ retrieved objects when Gaussian blur is applied to query object (hook) 
Table 3 Performance for VAE-induced and Hamming metrics given different level of salt and pepper noise as well as Gaussian blur for different kernel sizes

\begin{tabular}{|c|c|c|c|c|c|c|}
\hline \multirow[t]{2}{*}{ Dist. } & \multicolumn{3}{|l|}{ VAE } & \multicolumn{3}{|l|}{ Hamming } \\
\hline & $\mathcal{C}_{\text {free }}(\%)$ & $\mathcal{C}_{\text {cage }}(\%)$ & $Q_{c l}$ & $\mathcal{C}_{\text {free }}(\%)$ & $\mathcal{C}_{\text {cage }}(\%)$ & $Q_{c l}$ \\
\hline no dist. & 90.9 & 53.2 & 5.05 & 74.4 & 35.4 & 3.87 \\
\hline S\&P 0.005 & 91.1 & 52.8 & 4.94 & 74.9 & 34.5 & 3.86 \\
\hline S\&P 0.01 & 90.5 & 52.4 & 4.98 & 74.6 & 35.4 & 3.88 \\
\hline S\&P 0.05 & 83.4 & 45.0 & 10.12 & 71.8 & 35.0 & 3.81 \\
\hline S\&P 0.1 & 83.2 & 43.5 & 10.95 & 73.2 & 33.8 & 3.87 \\
\hline Gb $11 \times 11$ & 91.8 & 52.5 & 4.93 & 73.7 & 34.6 & 3.78 \\
\hline $\mathrm{Gb} 21 \times 21$ & 90.3 & 52.8 & 4.83 & 73.1 & 33.8 & 3.78 \\
\hline $\mathrm{Gb} 41 \times 41$ & 84.7 & 49.2 & 4.59 & 69.8 & 33.4 & 3.74 \\
\hline $\mathrm{Gb} 61 \times 61$ & 84.7 & 45.3 & 4.21 & 68.0 & 29.3 & 3.73 \\
\hline
\end{tabular}

Hamming distance appears to be better at capturing shape features that are relevant for cage acquisition task, it is the least efficient approach in terms of computation time. Furthermore, in our opinion, VAE-induced distance may be improved significantly if instead of using a general-purpose architecture we introduce task-specific geometric and topological priors.

\section{Limitations and Challenges for Future Work}

In this section, we discuss the main challenges of our work and the possible ways to overcome them.

\subsection{Data generation challenges}

One of the main challenges in this project is related to data generation: we need to densely sample the space of the caging tools' configurations, as well as the spaces of shapes of objects and caging tools. This challenge is especially significant when using the $P C$-general dataset, as the space of possible caging tools configurations is large.

While the experimental evaluation indicates that the chosen network architecture is able to achieve low MSE on previously unseen objects, in applications one may want to train the network with either a larger distribution of objects, or a distribution of objects that are similar to the objects that will be encountered in practice.

In Fig. 19, we illustrate how a lack of training data of sufficiently similar shapes can lead to poor performance of CageMaskNN and CageClearanceNN, for example, when only $1,10,100$, or 1000 objects are used for training. Similarly, even when the networks are trained on the full training dataset of 3048 objects, the subtle geometric details of the partial caging region cannot be recovered for the novel test object, requiring more training data and further refinement of the approach.

\subsection{Robustness under noise}

In the cage acquisition scenario, the VAE-induced and Hamming distances work directly with images, and hence can be susceptible to noise. To evaluate this effect, we generate salt and pepper noise as well as Gaussian blur and analyse the performance of the VAE-induced and Hamming metrics under four different noise levels $(0.005 \%, 0.01 \%, 0.05 \%, 0.1 \%)$ and four different kernel sizes $(11 \times 11,21 \times 21,41 \times 41,61 \times$ 61). ${ }^{8}$ Figure 20 shows the result of the top 3 retrieved objects for the hook object. Left column shows the query objects with respective disturbance. The next three columns depict the closest objects retrieved according to the VAE-induced metric, while the last three columns show the objects retrieved with Hamming metric.

Table 3 reports the performance with respect to finding collision-free configurations, configurations belonging to $\mathcal{C}_{\text {cage }}$, and their average values of $Q_{c l}$. The results are averaged over 500 novel objects. We can see that the VAEinduced metric is affected by strong salt and pepper noise as the number of generated collision-free and partial caging configurations decreases. Furthermore, the resulting $Q_{c l}$ of the generated partial cages increases, meaning it is easier to escape the cage. According to the experiment, the Hamming distance-based lookup is not significantly affected by salt and pepper noise. One explanation here may be that this kind of disturbance leads to a uniform increase of the Hamming distance for all objects. The Gaussian blur has a more negative effect on the Hamming distance lookup then the VAE-based lookup, as can be seen in the retrieved example objects in Fig. 20. Table 3 shows small decrease in the percentage of collision-free and partial caging configurations. Interestingly, the quality of the partial cages does not decrease.

\footnotetext{
8 Note that sigma is calculated using the standard OpenCV (Bradski 2000) implementation $(\sigma=0.3 \cdot((k s i z e-1) \cdot 0.5-1)+0.8)$.
} 


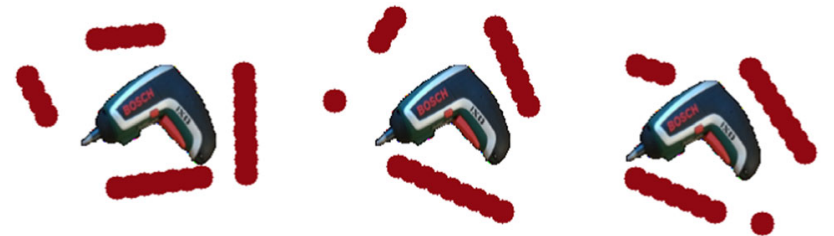

Fig. 21 Proposed partial cages using the VAE cage acquisition method. The novel object (hand drill) is feed into the cage acquisition and the best three cages from the closest object in the dataset are shown (in red) (Color figure online)

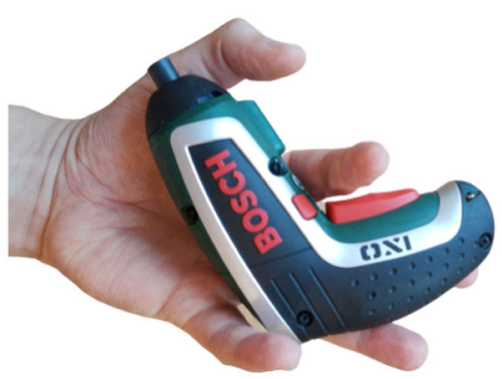

Fig. 22 An example for future partial caging in 3D. A complex object needs to be safely transported without the need to firmly grasp it

\subsection{Real World Example and Future Work}

As the VAE-framework just takes an image in order to propose suitable cages for a novel object, we showcase a concluding application example in Fig. 21 where a novel object (a hand drill) is chosen as input to the VAE cage acquisition. The image is preprocessed by a simple threshold function to convert it to a black and white image, next the closest object from the dataset are found by comparing the distances in the latent space of the VAE and the three best partial caging configurations are retrieved and applied to the novel object.

In the future, we would like to extend our approach to 3dimensional objects, As illustrated in Fig. 22, partial cages may be a promising approach for transporting and manipulating 3D objects without the need for a firm grasp, and fast learning based approximations to analytic or planning based methods may be a promising direction for such partial 3D cages. Furthermore, we would also like to to investigate the possibility of leveragingother caging verification methods such as Varava et al. (2018) for our approach.

Acknowledgements This work has been supported by the Knut and Alice Wallenberg Foundation, Swedish Foundation for Strategic Research and Swedish Research Council.

Funding Open Access funding provided by Royal Institute of Technology.

Open Access This article is licensed under a Creative Commons Attribution 4.0 International License, which permits use, sharing, adaptation, distribution and reproduction in any medium or format, as long as you give appropriate credit to the original author(s) and the source, provide a link to the Creative Commons licence, and indicate if changes were made. The images or other third party material in this article are included in the article's Creative Commons licence, unless indicated otherwise in a credit line to the material. If material is not included in the article's Creative Commons licence and your intended use is not permitted by statutory regulation or exceeds the permitted use, you will need to obtain permission directly from the copyright holder. To view a copy of this licence, visit http://creativecomm ons.org/licenses/by/4.0/.

\section{References}

Abadi, M., Barham, P., Chen, J., Chen, Z., Davis, A., Dean, J., et al. (2016). Tensorflow: A system for large-scale machine learning. In Symposium on Operating Systems Design and Implementation (pp. 265-283).

Bicchi, A., \& Kumar, V. (2000). Robotic grasping and contact: A review. In Proceedings 2000 ICRA. Millennium conference. IEEE international conference on robotics and automation. Symposia proceedings (Cat. No. O0CH37065) (Vol. 1, pp. 348-353). IEEE.

Bohg, J., Morales, A., Asfour, T., \& Kragic, D. (2013). Data-driven grasp synthesisa survey. IEEE Transactions on Robotics, 30(2), 289-309.

Bousmalis, K., Irpan, A., Wohlhart, P., Bai, Y., Kelcey, M., Kalakrishnan, M., et al. (2018). Using simulation and domain adaptation to improve efficiency of deep robotic grasping. In IEEE international conference on robotics and automation (ICRA) (Vol. 2018, pp. 4243-4250).

Bradski, G. (2000). The OpenCV library. Dr. Dobb's Journal of Software Tools, 25(11), 122-125.

Dai, B., \& Wipf, D. (2019). Diagnosing and enhancing vae models. arXiv: 1903.05789.

Gualtieri, M., Ten Pas, A., Saenko, K., \& Platt, R. (2016). High precision grasp pose detection in dense clutter. In 2016 IEEE/RSJ international conference on intelligent robots and systems (IROS) (pp. 598-605). IEEE.

Hamming, R. W. (1950). Error detecting and error correcting codes. The Bell System Technical Journal, 29(2), 147-160.

Johns, E., Leutenegger, S., \& Davison, A. J. (2016). Deep learning a grasp function for grasping under gripper pose uncertainty. In 2016 IEEE/RSJ international conference on intelligent robots and systems (IROS) (pp. 4461-4468). IEEE.

Kalashnikov, D., Irpan, A., Pastor, P. Ibarz, J., Herzog, A., Jang, E. Quillen, D., Holly, E., Kalakrishnan, M., \& Vanhoucke V. et al. (2018). Qt-opt: Scalable deep reinforcement learning for visionbased robotic manipulation. arXiv:1806.10293.

Kappler, D., Bohg, J., \& Schaal, S. (2015) Leveraging big data for grasp planning. In 2015 IEEE international conference on robotics and automation (ICRA) (pp. 4304-4311). IEEE.

Kinga, D., \& Adam, J. (2015). A method for stochastic optimization int. In Conference on learning representations (ICLR) .

Lembcke, S. (2013). Chipmunk 2d physics engine. Inver Grove Heights: Howling Moon Software.

Lenz, I., Lee, H., \& Saxena, A. (2015). Deep learning for detecting robotic grasps. The International Journal of Robotics Research, 34(4-5), 705-724.

Levine, S., Pastor, P., Krizhevsky, A., Ibarz, J., \& Quillen, D. (2018). Learning hand-eye coordination for robotic grasping with deep learning and large-scale data collection. The International Journal of Robotics Research, 37(4-5), 421-436.

Mahler, J., \& Goldberg, K. (2017). Learning deep policies for robot bin picking by simulating robust grasping sequences. In Conference on robot learning (pp. 515-524). 
Mahler, J., Liang, J., Niyaz, S., Laskey, M., Doan, R., Liu, X., Ojea, J. A. \& Goldberg, K. (2017). Dex-net 2.0: Deep learning to plan robust grasps with synthetic point clouds and analytic grasp metrics. arXiv:1703.09312.

Mahler, J., Pokorny, F. T., McCarthy, Z., van der Stappen, A. F., \& Goldberg, K. (2016). Energy-bounded caging: Formal definition and 2-d energy lower bound algorithm based on weighted alpha shapes. IEEE Robotics and Automation Letters, 1(1), 508-515.

Mahler, J., Pokorny, F. T., Niyaz, S., \& Goldberg, K. (2018). Synthesis of energy-bounded planar caging grasps using persistent homology. IEEE Transactions on Automation Science and Engineering, 15(3), 908-918.

Makapunyo, T., Phoka, T., Pipattanasomporn, P., Niparnan, N., \& Sudsang, A. (2012) Measurement framework of partial cage quality. In 2012 IEEE international conference on robotics and biomimetics (ROBIO) (pp. 1812-1816). IEEE.

Makita, S., \& Maeda, Y. (2008). 3d multifingered caging: Basic formulation and planning. In 2008 IEEE/RSJ international conference on intelligent robots and systems (pp. 2697-2702). IEEE.

Makita, S., Okita, K., \& Maeda, Y. (2013). 3d two-fingered caging for two types of objects: Sufficient conditions and planning. International Journal of Mechatronics and Automation, 3(4), 263-277.

McCarthy, Z., Bretl, T., \& Hutchinson, S. (2012). Proving path non-existence using sampling and alpha shapes. In 2012 IEEE international conference on robotics and automation (pp. 25632569). IEEE.

Pinto, L., \& Gupta, A. (2016). Supersizing self-supervision: Learning to grasp from 50k tries and 700 robot hours. In IEEE international conference on robotics and automation (ICRA) (Vol. 2016, pp. 3406-3413). IEEE.

Pipattanasomporn, P., \& Sudsang, A. (2006). Two-finger caging of concave polygo. In Proceedings 2006 IEEE international conference on robotics and automation, 2006. ICRA 2006 (pp. 2137-2142). IEEE.

Pokorny, F. T., Stork, J. A. \& Kragic, D. (2013). Grasping objects with holes: A topological approach. In 2013 IEEE international conference on robotics and automation (pp. 1100-1107). IEEE.

Rimon, E., \& Blake, A. (1999). Caging planar bodies by one-parameter two-fingered gripping systems. The International Journal of Robotics Research, 18(3), 299-318.

Rodriguez, A., Mason, M. T., \& Ferry, S. (2012). From caging to grasping. The International Journal of Robotics Research, 31(7), 886-900.

Saxena, A., Driemeyer, J., \& Ng, A. Y. (2008). Robotic grasping of novel objects using vision. The International Journal of Robotics Research, 27(2), 157-173.

Stork, J. A., Pokorny, F. T., \& Kragic, D. (2013a). A topology-based object representation for clasping, latching and hooking. In 2013 13th IEEE-RAS international conference on humanoid robots (humanoids) (pp. 138-145). IEEE.

Stork, J. A., Pokorny, F. T., \& Kragic, D. (2013a). Integrated motion and clasp planning with virtual linking. In IROS, Tokyo, Japan.

Szegedy, C., Liu, W., Jia, Y., Sermanet, P., Reed, S., Anguelov, D., et al. (2015). Going deeper with convolutions. In Proceedings of the IEEE conference on computer vision and pattern recognition (pp. $1-9)$.

Taha, A. A., \& Hanbury, A. (2015). An efficient algorithm for calculating the exact Hausdorff distance. IEEE Transactions on Pattern Analysis and Machine Intelligence, 37(11), 2153-2163.

Vahedi, M., \& van der Stappen, A. F. (2008). Caging polygons with two and three fingers. The International Journal of Robotics Research, 27(11-12), 1308-1324.

Varava, A., Carvalho, J. F., Pokorny, F. T., \& Kragic, D. (2018) Free space of rigid objects: Caging, path non-existence, and narrow passage detection. In Workshop on algorithmic foundations of robotics.
Varava, A., Welle, M. C., Mahler, J., Goldberg, K., Kragic, D. \& Pokomy, F. T. (2019) Partial caging: A clearance-based definition and deep learning. In 2019 IEEE/RSJ international conference on intelligent robots and systems (IROS) (pp. 1533-1540). IEEE.

Varava, A., Kragic, D., \& Pokorny, F. T. (2016). Caging grasps of rigid and partially deformable 3-d objects with double fork and neck features. IEEE Transactions Robotics, 32(6), 1479-1497.

Wan, W., \& Fukui, R. (2018). Efficient planar caging test using space mapping. IEEE Transactions on Automation Science and Engineering, 15(1), 278-289.

Zeng, A., Song, S., Yu, K.-T., Donlon, E., Hogan, F. R., Bauza, M., Ma, D., Taylor, O., Liu, M., \& Romo, E. et al. (2017). Robotic pick-and-place of novel objects in clutter with multi-affordance grasping and cross-domain image matching. arXiv:1710.01330.

Zhang, L., Kim, Y. J., \& Manocha, D. (2008). Efficient cell labelling and path non-existence computation using c-obstacle query. The International Journal of Robotics Research, 27(11-12), 1246-1257.

Publisher's Note Springer Nature remains neutral with regard to jurisdictional claims in published maps and institutional affiliations.

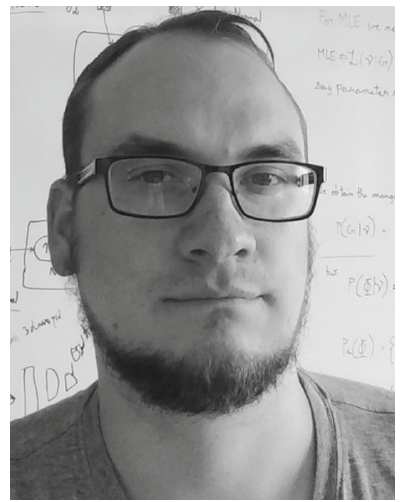

Michael C. Welle received his MSc degree in Systems, Control and Robotics from KTH Royal Institute of Technology, Stockholm, Sweden in 2018. He is currently a Ph.D. student at KTH Royal Institute of Technology, Stockholm, Sweden. $\mathrm{He}$ is interested in different representations of rigid and deformable objects in Robotics and Machine Learning.

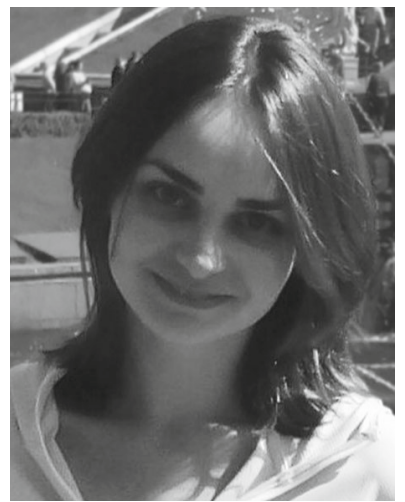

Anastasiia Varava received her MSc degree in Computer Science from the University of Nice Sophia Antipolis, France, in 2014. She received her $\mathrm{PhD}$ degree from KTH Royal Institute of Technology, Stockholm, Sweden in 2019. She is interested in geometric and topological applications to Robotics and Machine Learning. 


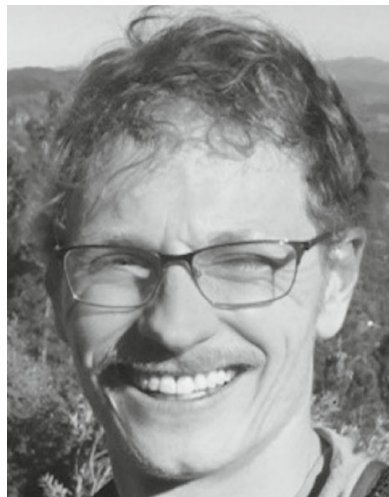

Jeffrey Mahler (M'18) received the B.S. degree (Hons.) in electrical engineering from the University of Texas at Austin, Austin, TX, USA, in 2013. He is currently pursuing the Ph.D. degree in electrical engineering and computer sciences (with a specialization in robotics and artificial intelligence) with the University of California at Berkeley, Berkeley, CA, USA. He co-founded the 3 D scanning company Lynx Laboratories in 2012, which was acquired by Occipital in 2015 . His research interests include robust robot grasping, deep learning for robot perception, and imitation learning. Mr. Mahler was awarded the the National Defense Science and Engineering Graduate Fellowship in 2015.

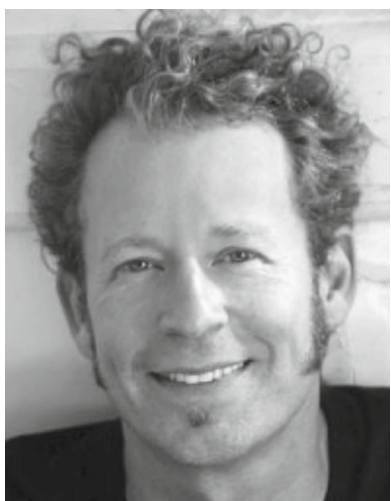

Ken Goldberg (F'18) received the $\mathrm{Ph} . \mathrm{D}$. degree in computer science from Carnegie Mellon University, Pittsburgh, PA, USA, in 1990. He is the William S. Floyd Distinguished Chair of Engineering and the Chair of the Industrial Engineering and Operations Research Department, University of California at Berkeley, Berkeley, CA, USA, with appointments in electrical engineering, computer science, art practice, and the School of Information. He has authored more than 250 refereed papers and holds eight U.S. patents. Dr. Goldberg was a recipient of the NSF Presidential Faculty Fellowship in 1995, the Joseph Engelberger Award in 2000, and the IEEE Major Educational Innovation Award in 2001. He is the Founding Co-Chair of the IEEE Technical Committee on Networked Robots and the Founding Chair of the (T-ASE) Advisory Board. He served as the Editor-in-Chief for the IEEE TRANSACTIONS ON AUTOMATION SCIENCE AND ENGINEERING from 2011 to 2016 and served two terms from 2006 to 2009 as the VicePresident of Technical Activities for the IEEE Robotics and Automation Society.

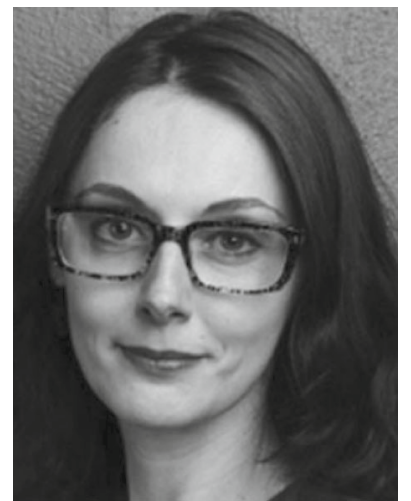

Danica Kragic (SM'12) received the M.Sc. degree in mechanical engineering from TU Rijeka, Rijeka, Croatia, in 1995 and the Ph.D. degree in computer science from KTH Royal Institute of Technology, Stockholm, Sweden, in 2001. She is a Professor with the School of Computer Science and Communication, KTH Royal Institute of Technology. Her research interests include computer vision and robotics. Prof. Kragic is a member of the Swedish Royal Academy of Sciences and the Swedish Young Academy. She received the 2007 IEEE Robotics and Automation Society Early Academic Career Award.

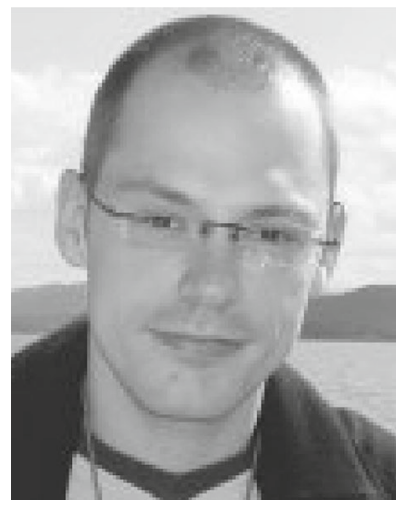

Florian T. Pokorny (M'18) received the B.Sc. degree in mathematics from the University of Edinburgh, Edinburgh, U.K., in 2005 and completed Part III of the Mathematical Tripos with the University of Cambridge, Cambridge, U.K., in 2006. He then received the Ph.D. degree in mathematics from the University of Edinburgh in 2011. In 2011, he joined the KTH Royal Institute of Technology, Stockholm, Sweden, as a PostDoctoral Researcher. From 2015 to 2016, he conducted PostDoctoral Research with the AMPLab and the Berkeley Automation Science and Engineering Laboratory, University of California at Berkeley, Berkeley, CA, USA, under the supervision of Prof. K. Goldberg. He is currently an Assistant Professor with the Robotics, Perception and Learning Lab, KTH Royal Institute of Technology. His research interests include robotic manipulation, machine learning, and topological data analysis. 